\title{
Adaptive Front Lighting System Using CCD
}

\author{
Prajakta Vikas Adhav, Prof. S. A. Shaikh \\ Electronics and Telecommunication, Pravara Rural Engineering College Pravaranagar, India. \\ Electronics and Telecommunication, Pravara Rural Engineering College Pravaranagar, India.
}

\begin{abstract}
This paper analyzed features of headlamps of vehicle when turning the corner. When vehicle is driven on curved road headlamps turns as per vehicle body hence it has limited illumination. The adaptive front lightning system (AFS) improves visibility during night time driving. This project developed a new type of AFS (adaptive front-lighting system) based on CCD (charge-coupled device) which was better than traditional AFS. The main aim is to find curvature of road ahead in advance before turning the corner so as to adjust turning angle and steering accordingly. This new AFS used CCD image sensor to detect information about the corner and then sent curvature radius to control unit in advance. Meanwhile, accurate rotation angle of headlamps would calculated by control unit through curvature radius, and send it to servomotor to adjust headlamps to get the lighting beam which was suitable for the corner. By using this way, it could avoid "blind spot" caused by the fixed lighting area when coming into the corner, and improve driving safety.
\end{abstract}

Keywords: photometric characteristics, adaptive front-lighting system, charge-coupled device, curvature radius, horizontal rotation angle, modeling and simulation.

\section{Introduction}

In recent years, more and more vehicles are used in our daily life, consequently, more and more traffic accidents happen. According to survey out of all accidents $82 \%$ of traffic accidents happened at night because of bad illumination condition. Also it shows that the number of serious accidents at night is about 1.5 times of the day, and $60 \%$ of accidents happened at corners with poor lighting. The main reason behind it is blind spot. Blind spot is caused by fixed lightning area. The vehicle turning the corner at night is unable to adjust the range and direction of optical axis and visual "blind spot" comes out inside corner. To solve this problem the "adaptive front-lighting system", namely AFS, has came into picture. The AFS controls the aiming direction and lightning distribution of te low beams according to the amount of turn applied to the steering wheel during turning. According to weather conditions, road and driving conditions different light types are required. Adaptive front-lighting system is an active safety system which can change automatically two or more kinds of light types for different external factors. However, to some extent, although the currently existing AFS can improve driving safety at the corner, the system has some limitations. The main disadvantages are lag and lower precision, which are caused by reaction time that the driver needs to turn steering wheel when seeing the front corner. At this time, if obstacles are ahead and the driver is inadequate preparation, it's easy to cause accidents. So, to avoid limitations of traditional AFS this paper developed improved version of AFS. Traditional AFS has played an important role in safety fields, but at the same time its limitations could not be ignored. Based on this point, an adaptive front-lighting system based on CCD which was better than the traditional one has developed. Because of use of CCD it guides driver when turning into the corner. This new kind of AFS uses CCD image recognition technology.CCD captures the image of corner from certain distance before turning the corner. And then it adjusts different angles of dipped headlights in advance according to the corner information collected. So, in this case "blind spot" is avoided as headlamps are adjusted in advance as per corner information. This way, it can adapt to corner condition in advance through CCD with canceling calculating corner radius by steering wheel angle sensor. As a result, it makes up for traditional AFS system's limitations of obviously lag and lower headlamp rotation angle, and realizes active rotation of headlamps. So the AFS corner lighting system based on CCD image recognition technology is a real sense of active safety system.

\section{Adaptive Front Lightning System}

The most important active safety functions of vehicles are headlamps. Headlamps performance has steadily improved as automobile age increases, with great disparity between daytime and nighttime traffic fatalities. so the light distribution of headlamps should be able to fulfill all needs such as, no glare, sufficient illumination, enough range of lightning to avoid accidents. Beginning in the 2000s there was resurgence in interest in the idea of moving the headlight beam in response not only to vehicular steering and suspension, but also to ambient whether and visibility conditions, speed, and road curvature. The task force under the EUREKA organization began working to develop design and performance specification for what is called Adaptive Front Lightning System (AFS). [1] 


\subsection{Major Functions provided by AFS are as follows,}

- Town passing beam (Class V): At speeds below $50 \mathrm{~km} / \mathrm{h}$, town light provides a wider light distribution at reduced range, helping drivers to more clearly see pedestrians on the edge of the road.

- Basic/Country passing beam (Class C): The basic light illuminates the left- and right-hand edges of the road more brightly and widely than the conventional low beam. It is usually activated at speeds between 50 and $100 \mathrm{~km} / \mathrm{h}$.

- Motorway passing beam (Class E): Motorway light improves vision on highways and expressways. From $100 \mathrm{~km} / \mathrm{h}$, this beam illuminates the roadway significantly further ahead and focuses more on the left-hand edge of the road. The motorway light switches on automatically at speeds greater than 100 $\mathrm{km} / \mathrm{h}$.

- Wet-road passing beam (Class W): This beam is activated when the rain sensor detects precipitation or the windshield wipers are on for 2 minutes or more. The edges of the road are more strongly illuminated for better orientation to the guiding lines.

- Static cornering light: Static cornering light helps during maneuvers in dark access roads. At speeds of up to $40 \mathrm{~km} / \mathrm{h}$, one additional cornering light comes on when the indicator is actuated or the steering wheel turned through about 90 degrees to the right or left.

- Dynamic swiveling and leveling:

- Leveling: The AFS adjusts the direction of the headlight (projector) vertically. Adjusting the pitch angle of the headlight according to static vehicle load transfer is called static leveling, while adjusting headlight pitch angle according to dynamic vehicle load transfer is known as dynamic leveling.

- Swiveling: The AFS swivels the headlights horizontally by judging the input from the steering angle sensor and the speed of the car. A curve rate at up to 15 degrees is provided by this system and obstacles become more easily visible. [2]

\subsection{Light Environment of Headlamps without AFS}

In our daily life, while using vehicles at night we face problem of blind spot when turning the corner.Headlamps are parallel to body of vehicles so, for example while turning to left most of the light beam is projected on right and left projected part becomes narrower. Therefore it will be unable to detect obstacle in left front.And blind spot comes into picture as shown in figure. It is shown in figure 1 that how blind spot occurs while turning in the corner. To avoid problems associated with traditional AFS new type of AFS based on CCD is developed. It use CCD image sensor to collect information of curve road in advanced so that headlamps get adjust automatically accordingly.

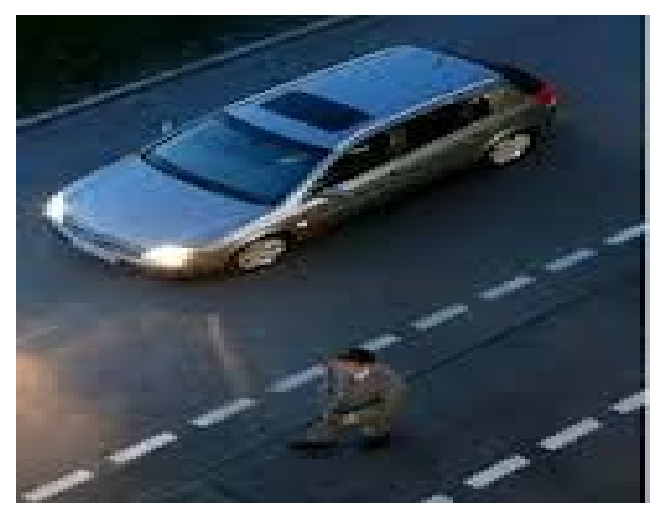

Figure 1: Light environment of headlamps without AFS

\subsection{Light Environment of Headlamps with AFS}

Adaptive Front Lightning System is one part of active safety system of middle and high end passenger cars providing an optimized vision to the driver at night time and other poor sight conditions of road by adapting headlight angle and intensity. Firstly, the existing vehicle adaptive front-lighting system (AFS) can detect driver's steering operation and state of traffic condition through sensors. By this way, it can get parameters like steering wheel angle, velocity, and body height information which are needed later. By using this information rotation angle of headlamps is calculated and are rotated according to corner. Figure 2 shows vehicle with AFS. It has better light illumination and it overcomes the disadvantages of vehicle without AFS. [3] 


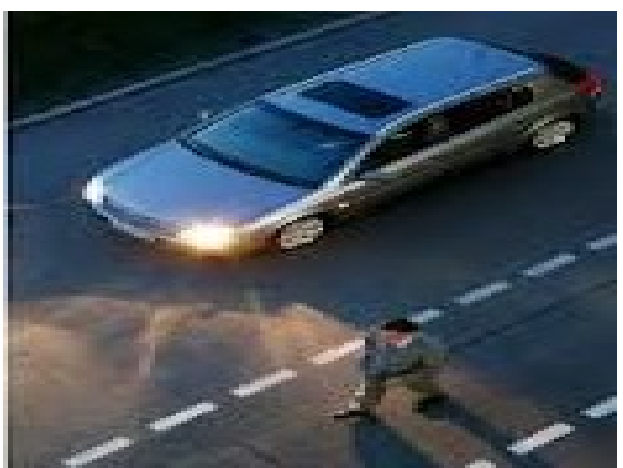

Figure 2: lighting area of headlamps with AFS when driving in the corner

\section{Working Principle Of Afs Corner Lighting System Based On Ced}

To avoid problems associated with traditional AFS new type of AFS based on CCD is developed. It use CCD image sensor to collect information of curved road in advanced so that headlamps get adjust automatically according to it.

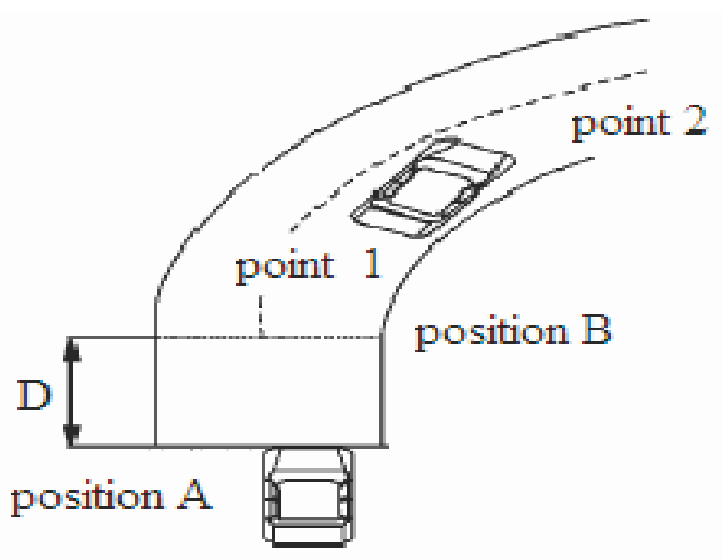

Figure 3: Schematic diagram of driving into the corner

Above figure shows the working principle of vehicle having AFS with CCD. When vehicle comes to position A it detects corner ahead and CCD sensor captures the image of corner ahead and by using MATLAB processing rotation angle of headlamps is calculated. Now, when vehicle reach at position B headlamps are already adjusted in advance according to rotation angle and it can turn easily without any problem.

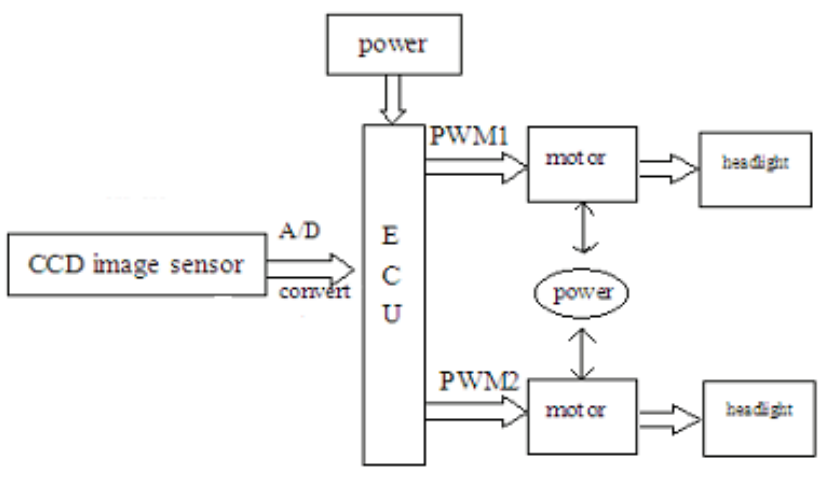

Figure 4: Block Diagram

Figure below shows schematic of vehicle installed with new AFS that is AFS safety system using CCD image sensor. Rotation angles are different for different directions in which vehicle turns. When vehicle turns left light is focused on left part of the road using left headlamp and for right part right is focused on right part of road.

Figure shows a vehicle driving on the straight lane, which is point $\mathrm{A}$ in figure, headlamps beam is parallel to lane and there is enough light to illuminate the road ahead. When vehicle will go into the corner, B 
point as shown, it adjusts the beam in advance through AFS system to adapt to the front corner, and now vehicle comes into the corner, there no longer appears visual "blind spot" inside corner. When going out of the corner, C point, according to the situation of the road ahead, beam is adjusted to be parallel to lane and adaptive to driving straight again.

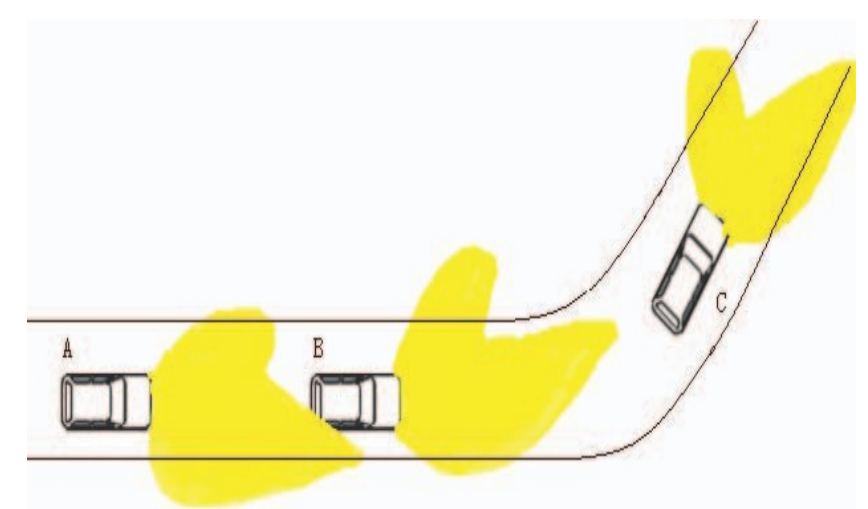

Figure 5: Schematic diagram when the vehicle with this new AFS

Vehicle with this new AFS when turning left. This new AFS system detects the velocity of vehicle through speed sensor, so as to get stopping sight distance $\mathrm{S}$, obtains curvature radius $\mathrm{R}$ through the CCD image sensor, so as to get a rotation angle $\theta$ in horizontal direction, and then get lighting beam which is adaptive to corner through actuator motor (rotary motor) rotating headlamps in horizontal direction. [4] [5]

\section{Software Used}

Here, Matlab is used for building mathematical model of this system. Image processing hardware will be used as sensor for detecting radius of curvature of road. Camera of $640 * 480$ resolution and $16 \mathrm{M}$ color depth will be used as input. Matlab will configure this camera for manual trigger. When image is to be captured Matlab code will trigger the camera and obtain live image from camera. This image will go under series of operations like preprocessing, color conversions, segmentation, feature extraction and decision, and this result of road curvature will be sent to ARM11 by serial port. Serial port is initialized with 8 bit data bits and 9600bps baud rate.

After receiving result from Matlab, microcontroller will calibrate if in angle and will change the pwm signal fed to servo motor. Camera and horizontal servo motor will make on sensor-actuator loop. Figure.6 shows the flowchart of this project. It shows how image processing will going to be happen. First camera properties are set. Then camera is triggered and we will get the image in RGB forma. After this process image will be displayed. Then image get converted from RGB format to gray format. Then image is cropped from complete view of image. From image closer contour of road will be find. In this we are going to use Hough transform for image processing. After that Hough transform will be find. From Hough transform rotation angle for the turn will be calculated. If road is turning towards right then $\mathrm{R}$ is send to robot and if road is turning left then $\mathrm{L}$ is send to road. In this way Matlab processing is use in this project for image processing to find rotation angle of road so that vehicle can done adjustment of headlamps in advance to avoid obstacles. [6] [7] 


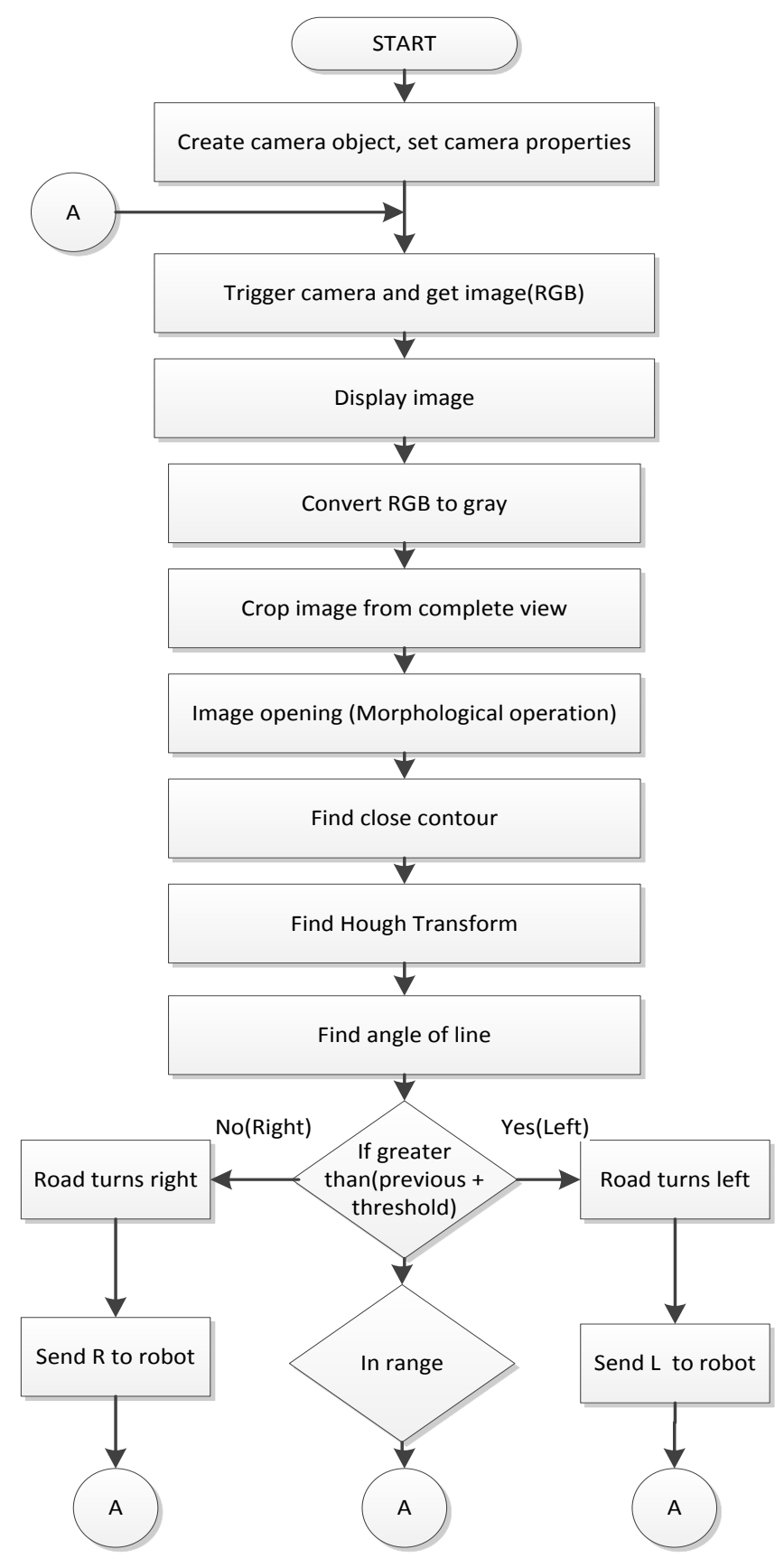

Figure 6: Image processing flowchart

\section{Applications}

- In cars to avoid accidents at night time. When driving on the winding road, AFS with CCD will change the lighting pattern to compensate for the curvature of the road to help enhance night visibility.

- It can be modified and used for movable surveillance cameras.

- Can be used for robotic vision and robotic ARM movement.

- It is very responsive and fast system.

\section{Advantages}

- It will definitely help to improved road illumination.

- More range vision in a curve compared to traditional AFS.

- The arrival of this new technology will give opportunities for new suppliers to enter the market.

- It provides Safety during street crossings, at junctions and cornering. 


\section{Conclusion}

As discussed above, at present, existing AFS is not sufficient to overcome problems of driving at corner during night time. It has disadvantages like lag and precisions, to avoid this disadvantages this paper put forward an advanced AFS using CCD image sensor. Traditional AFS has greater error in getting curvature radius. This new AFS provides curvature radius information superior than traditional.

CCD sensor is used to detect real time information of curved road. In this movement of light beam is earlier than turning steering wheel by driver. Meanwhile, the light beam of this new AFS can guide driver's sight actively to reflect the active idea of AFS, and furthermore to improve the characteristic of intelligent and initiative of AFS control system. On the other hand, AFS system based on CCD image recognition technology proposed in this paper is not only for corner lighting, but also can provide comprehensive information recognition in the future because of fast acquisition ability of CCD image sensor. So it's probable to invent a comprehensive AFS system which can be suitable for complex road conditions including road surface water, corner, highway, rural road and urban road and so on. This kind of AFS system has earlier perception ability to obtain complex road conditions, and adjust corresponding light distribution pattern in advance.

Therefore, the CCD image recognition technology with its high sensitivity, high earthquake resistance and low cost will be a future development direction of AFS.

\section{Acknowledgment}

It is a matter of gratification for me to pay my respects and acknowledgements to all those who have imparted knowledge and helped me to complete my paper.

I would first like to acknowledge the great contribution and support I have received in this endeavor from Guide Prof.S.A.Shaikh His in depth guidance and inspiration for will be of great help to tackle any kind of problems likely to be met in the future.

I express my sincere thanks to Head of Dept Prof.S.M.Turkane and M.E. Co-ordinator Prof S. G Galande for their valuable guidance.

\section{References}

[1] Research of Modeling and Simulation on Adaptive FrSystem for Corner Based on CCD. Fengqun Guo, Hui Xiao, Shouzh T. Aoki, H. Kitamura, K. Miyagawa, and M. Kaneda, (1997). "Development of active headlight system," (SAE Technical Paper Series No. 970650). Warrendale, PA: Society of Automotive Engineers, 1997.

[2] H. Hogrefe and R. Neumann, "Adaptive Light Pattern - A new way to improve Light Quality," SAE 970644, 1997.

[3] E. Groh, "Intelligent Lighting," SAE 970645, 1997.

[4] H. Hogrefe, "Adaptive front lighting systems for optimum illumination of curved roads, highway lanes and other driving situations," SAE 2000-01-0431. Warren dale, PA: Society of Automotive Engineers, 2000.

[5] K. Ishiguro and Y. Yamada, "Control technology for bending mode AFS," SAE 2004-01-0441. Warren dale, PA: Society of Automotive Engineers, 2004.

[6] M. Cejnek and D. Mikus, “Advanced Adaptive Front lighting system,” Proceedings of ISAL 2005 Symposium, Paper A11.6 (2005), pp. 333-342, 2005.

[7] C. Falcon, "Stepper Motors Assist Adaptive Headlights," Retrieved August 5, 2007, from http://www.automotivedesignline.com 